REVIEW PAPER

\title{
Personality and genes: remarks from a biological perspective
}

\author{
Grzegorz Wegrzyn \\ Department of Molecular Biology, University of Gdansk, Gdansk, Poland
}

\begin{abstract}
Although there is no doubt that genes' functions influence human personality, years of studies provided no clear picture on regulation of particular traits by specific genes. In this article, an overview of the complexity of the system of genetic control of personality is presented, and the level of complications of biological processes operating in this system is underlined. The methodology of studies devoted to determine effects of genes on personality traits is discussed, and limitations of various methods in such studies are indicated. Finally, suggestions for further research are listed and commented on. It is likely that to increase the level of our understanding of
\end{abstract}

genetic mechanisms that modulate human personality, researchers conducting further studies will have to focus on using large sample sizes, performing independent replications, considering experiments on animal models, integrating cross-cultural data and epigenetic measures, and performing interdisciplinary experiments which combine methods of various disciplines, such as biology and psychology.

KEY WORDS

gene function; genetic regulation; human personality; methods of genetic studies

CORRESPONDING AUTHOR - Grzegorz Węgrzyn, Department of Molecular Biology, University of Gdansk, 59 Wita Stwosza Str., 80-308 Gdansk, Poland, phone: +48 5852360 24, fax: +48 5852355 01, e-mail: grzegorz.wegrzyn@biol.ug.edu.pl AUthors' CONTRIBUtion - A: Study design - B: Data collection · C: Statistical analysis · D: Data interpretation .

E: Manuscript preparation · F: Literature search · G: Funds collection to Cite this ARTICLE - Węgrzyn, G. (2014). Personality and genes: remarks from a biological perspective. Current Issues in Personality Psychology, 2(3), 133-140. 


\section{BACKGROUND}

Personality is undoubtedly one of the most complicated features of humans, particularly from the biological point of view. This is due to the extremely complex structure and functions of the human brain, the organ whose development gave the human a very special position among all living creatures on Earth. For a biologist, it is clear that personality depends on brain function, and the brain structure (which is the basis for its functions) depends on the functions of many genes. Therefore, studies on mechanisms of personality are particularly difficult and complicated. Until relatively recently, the subject of human personality, as a research field, was dominated by classical psychological and sociological studies. However, in recent years, biological (broadly speaking) methods have became more and more popular in the process of investigation of this subject. Since every human, as a biological entity, develops from a single, undifferentiated cell - the zygote - for any biologist it must be obvious that development of all features of the further human organism depends directly or indirectly on the functions of certain genes. This does not mean that genes determine everything, as it is also obvious that environmental conditions (including social interactions) have a great influence on development, features and functioning of humans. Nevertheless, it would be very hard to imagine that development of human personality could not be influenced by the functions of many genes. Such a scenario is so unlikely that it is considered impossible by the vast majority, if not all, biologists, sociologists and psychologists. The basic question remains, however, whether genes are necessary only to direct the formation of the brain as a biological organ (one of many in the human body) and to retain its basic biological functions, or any human behavior is determined by genes. As is usual in biology, there is also a wide spectrum of intermediate possibilities between these two extremes.

Since a couple of decades ago, when biological techniques allowed us to study human genes in a broader way than only analyzing certain sequences and basic functions, the interest in considering a role for genes in formation of human personality has increased gradually. This can be exemplified by studies on the role of human genotype in the cycle of violence in maltreated children (Caspi et al., 2002), the subsequent finding that the influence of life stress on depression can be moderated by a polymorphism (occurrence of more than one form, called alleles, of a particular gene in the population) in the 5-HTT gene (Caspi et al., 2003), and investigations following those pioneering studies (reviewed by Buades-Rotger \& Gallardo-Pujol, 2014). Now, there are many reports in the literature addressing the influence of dysfunctions or polymorphisms of various genes on formation of and changes in human personality. On August 16, 2014, the query: "personality AND gene" gave 3819 records in the PubMed database (http:// www.ncbi.nlm.nih.gov/pubmed/?term=personality+AND+gene).

The aim of this article is not, however, to present particular issues which were addressed in those reports. Rather, an overview on the current stage of research on genetic aspects of human personality, and recently published opinions by different researchers, as well as the author's own opinion on possibilities for further directions of such studies, will be presented.

\section{HOW TO STUDY GENETICS OF PERSONALITY?}

The classical genetic attempt to study effects of genes on any biological process is to find a mutated (dysfunctional or at least changed) form of the tested gene, and to investigate the influence of the mutation on the organism. Another possibility, particularly if one is searching for an unknown gene, is to find a mutant (changed) organism, and to perform genetic analysis to identify a gene whose dysfunction causes the observed effects. In the case of most studied biological species, a geneticist can (in a more or less complicated manner) create mutant organisms, bearing mutations in desired gene(s), and investigate their features. For obvious reasons, it is not possible in human genetics. Therefore, it is not surprising that the vast majority of studies addressing roles of genes in personality were based on employment of either naturally occurring disturbances in traits or psychiatric cases. Below, only some very recent reports are mentioned, just to exemplify such kind of studies.

Studies on the oxytocin receptor gene (OXTR) indicated that a particular polymorphism in this gene may influence the link between early family quality and later borderline personality disorder symptoms (Hammen, Bower, \& Cole, in press). Another work on the same gene revealed that the genetic variants (alleles, polymorphisms) associated with greater risk for higher borderline symptomatology dependent on maltreatment experiences were opposite in girls compared to boys with borderline personality disorder (Cicchetti, Rogosch, Hecht, Crick, \& Hetzel, 2014). This disorder can be moderated not only by mutations causing changes in products (usually proteins) of certain genes, but also by genetic changes resulting in decreased or increased efficiency of expression of genes, which sounds reasonable as various amounts of particular proteins may be responsible for various phenotypes. An example of this phenomenon has been published recently, where epigenetic changes (in the form of DNA methylation levels) in the promoter region of the $\mathrm{NR} 3 \mathrm{C} 1$ gene were correlated with clinical severity of this disease 
(Martin-Blanco et al., 2014). The current state of our knowledge on the genetic background of borderline personality disorder has been excellently reviewed in a recent article by Amad, Ramoz, Thomas, Jardri, and Gorwood (2014).

Another recent review article (Buades-Rotger \& Gallardo-Pujol, 2014) summarized studies published to date on the role of the MAOA gene, coding for monoamine oxidase $\mathrm{A}$, in the antisocial response to adversity. The MAOA gene product is an enzyme degrading neurotransmitters such as serotonin or dopamine. Thus, different activities of this protein, arising from expression of different alleles of MAOA, may influence various processes in the human brain, likely affecting personality. Buades-Rotger and Gallardo-Pujol (2014) also provided various examples of genes whose dysfunctions were evidently correlated with serious problems of different diseases and addictions (see references therein).

The advance of investigations of mutations causing severe behavioral effects, such as personality deviations or psychiatric diseases, is that results of particular genetic changes are obvious and unequivocal. However, in processes of extreme complexity, such as development of personality, the results of such studies may be easily over-interpreted. Generally, mutations in protein-encoding genes cause changes in functions of the gene products (proteins), which in cases of enzymatic proteins usually result in their lower or higher activities. This results in particular metabolic changes, leading to modified levels of certain compounds in the organism. If the compound is a hormone or a neurotransmitter, one might easily imagine effects on functions of the brain, and thus on behavior, and further on personality. Nevertheless, it is necessary to remember that primary changes in metabolism, resulting from dysfunction (or changed function) of one enzyme, may cause secondary and tertiary changes; thus, the final effects of tested mutations may be indirect, rather than direct.

Such a situation can be exemplified by many inherited metabolic diseases, in which problems with behaviors of patients are secondary or tertiary effects of perturbations in metabolism of compounds that are not directly involved in the processes of the transfer of signals by neurons. For instance, dysfunctions of various enzymes responsible for degradation of different compounds in lysosomes are causes of the group of disorders known as lysosomal storage diseases, among which many are characterized by (beside somatic problems) severe neurological and behavioral changes (Bellettato \& Scarpa, 2010). Among them there are mucopolysaccharidoses, inherited (in a recessive, either autosomal or X-linked, manner) diseases arising from a lack or a severe deficiency in activity of one of the enzymes involved in degradation of complex sugars, known as glycosaminoglycans (Wraith, 2013). Very severe and very different perturbations in personality of patients suffering from these diseases can be observed, depending on which biochemical step in the pathway of degradation of glycosaminoglycans is affected, and what is the level of residual activity of the affected enzyme in the patient's cells (reviewed and discussed by Węgrzyn et al., 2010). Mucopolysaccharidoses are classified according to dysfunctions of enzymes whose absence or significantly decreased activity results in inefficient degradation of glycosaminoglycans and accumulation of particular metabolites (partially degraded sugars). As discussed previously (Węgrzyn et al., 2010), even small chemical differences in the storage material might result in significant final effects on behavior of patients. Those suffering from mucopolysaccharidosis type II, and particularly MPS III, develop an aggressive-like personality. Children with mucopolysaccharidosis type II are often overactive, but this symptom is especially severe in patients with type III, who are usually described as hyperactive. These patients may become unexpectedly angry or may laugh without any reason. Contrary to them, children suffering from type I are generally placid, gentle and calm. Furthermore, they are often over-careful, while patients with type III appear to ignore any danger, and their behavior suggests intensive action without any particular sense.

Detailed analysis of available experimental data led to conclusions that accumulation of the partially degraded glycosaminoglycans is not the primary cause of neurological and behavioral problems, which might also be recognized as changes in personality, of patients suffering from mucopolysaccharidoses. Rather the battery of secondary and tertiary factors and processes, appearing as a consequence of the storage, is responsible for changes in brain functions, leading to the effects described above (Jakóbkiewicz-Banecka et al., 2014). Therefore, although mutations in genes coding for enzymes responsible for degradation of glycosaminoglycans (e.g. $\alpha$-L-iduronidase, iduronate-2-sulfatase, and several others) result in very specific changes in personality of patients, it is unlikely that slight modulations of activities of these enzymes could significantly modify the personality in healthy subjects. If so, one can ask if similar phenomena could occur as effects of mutations found in patients suffering from psychiatric diseases or personality perturbations. Thus, can we conclude that genes in which such mutations occurred are directly involved in formation of specific personality?

Because of the uncertainty described above, the use of other methods (apart from genetic analysis of disorders with changes in personality) appears necessary. As in other studies in the field of human genetics, animal models would be very useful, particularly since it is possible to perform experiments with them which could not be conducted with human 
beings due to ethical aspects. In fact, various animal models were used in such studies, not only primates, but also mice or even zebra fish, which can be exemplified by recently published reports (Schwartz et al., 2014; Laplana et al., in press). Experiments on animal models give an excellent opportunity to understand molecular mechanisms of functions of particular genes and their products, with special importance of those evolutionarily conserved in vertebrates or at least mammals. However, the question remains, to what extent could the animal models reflect biological processes characteristic for human personality? I am afraid that no precise answer can be given to this question at the current state of our knowledge.

The third group of possible methods for investigation of genes involved in personality is based on genetic studies of healthy subjects with different personality characteristics. Examples of recently published studies employing such a methodology include demonstration of the correlation between one of alleles of the ACP1 gene and reduced level of extraversion (Napolioni et al., 2014), involvement of the CRHR1 gene polymorphism in modulation of the risk of excessive alcohol consumption in response to negative emotions (Glaser et al., 2014), and modulation of cooperativeness, self-directedness and reward dependence by different alleles of genes ADRA2B, HTR2A, and SHBG, respectively (Calati et al., 2014). Another work indicated that despite some predictions, no correlation between different alleles of the APOE gene and personality could be found (Montag et al., 2014). Although it might appear that this methodology may be an optimal way to find relations between genes and personality, the major problem that appears is that results of such studies are extremely difficult to interpret. This is mainly due to the fact that personality is definitely not a monogenic feature, i.e. it is not encoded by a single gene. On the contrary, it is likely that hundreds or thousands of genes contribute, to a varying extent, to development and expression of personality. Therefore, small differences in activities and functions of each of these genes may contribute to the whole picture, expressed as the phenotype of specific personality. These form a network of interactions and interdependences that is impossible to decipher when studying effects of minor changes in one or a few gene(s). Therefore, any conclusions based on studies of genetic polymorphisms affecting personality must be very careful, as what we actually observe may be a very indirect effect or a coincidental result.

\section{THE LEVEL OF COMPLEXITY OF THE SYSTEM}

The high biological and/or genetic complexity of human personality was mentioned several times in pre- ceding paragraphs. However, one may ask what it means in comparison to other biological processes or systems for which genetic regulation have been relatively well established. Here, I will provide an example of one of the simplest regulatory systems that has been investigated for years, and whose regulation, based on activities of genes and their products, is relatively well understood. The model organism is bacteriophage $\lambda$, a virus infecting Escherichia coli, a bacterium living normally as a component of mammalian intestinal flora. Bacteriophage $\lambda$ and humans are at two extremes of complexity of structure and function of living entities. Genetic material of this virus consists of 48,502 base pairs, relative to about $6,000,000,000$ base pairs included in the DNA of each human diploid cell. Bacteriophage $\lambda$ DNA contains about 50 genes, while the number of human genes is estimated at about 25,000. Obviously, the virus has no personality, understood as a human feature. However, in its life cycle, it has one major decision to be made. Namely, there are two alternative pathways of bacteriophage $\lambda$ development after infection of a bacterial cell. The first option is to use the cellular machinery to produce viral nucleic acids and proteins, formation of progeny bacteriophages, and cell lysis with liberation of newly produced viruses. The second, alternative option is to integrate viral DNA into host genetic material, and survive silently as part of the bacterial cell (this process is called lysogenization). Depending on environmental conditions, either the lytic or lysogenic pathway may give the phage a better chance to survive and multiply efficiently. Therefore, the decision whether to lyse the host cell or to lysogenize is fundamental for the bacteriophage propagation. One may say that the whole "personality" of bacteriophage $\lambda$ is based just on making this one crucial decision.

How making the lysis-versus-lysogenization decision (i.e. bacteriophage "personality") is regulated by genes is depicted in Figure 1. This figure represents a simplified fragment of bacteriophage $\lambda$ DNA with indicated genes and their products involved in this regulation, some host (E. coli)-encoded proteins taking part in this process, and a network of interactions leading to making the final decision. My intention of showing this figure is not to describe the details of the regulatory process, but rather to demonstrate the level of complexity of the genetic regulation of a simple biological process. One can try to imagine the level of complication of a regulatory network of human personality, where thousands of genes (rather than several genes, like in bacteriophage $\lambda$ ) are involved, and millions of decisions (rather than just one, like in bacteriophage $\lambda$ ) are made. It is important to note that in purely biological systems, like the bacteriophage life cycle, the term "decision" is a personification, as "decision" means entering one of the alternative pathways (biochemical or physiological) rather than a kind of making a conscious choice. 


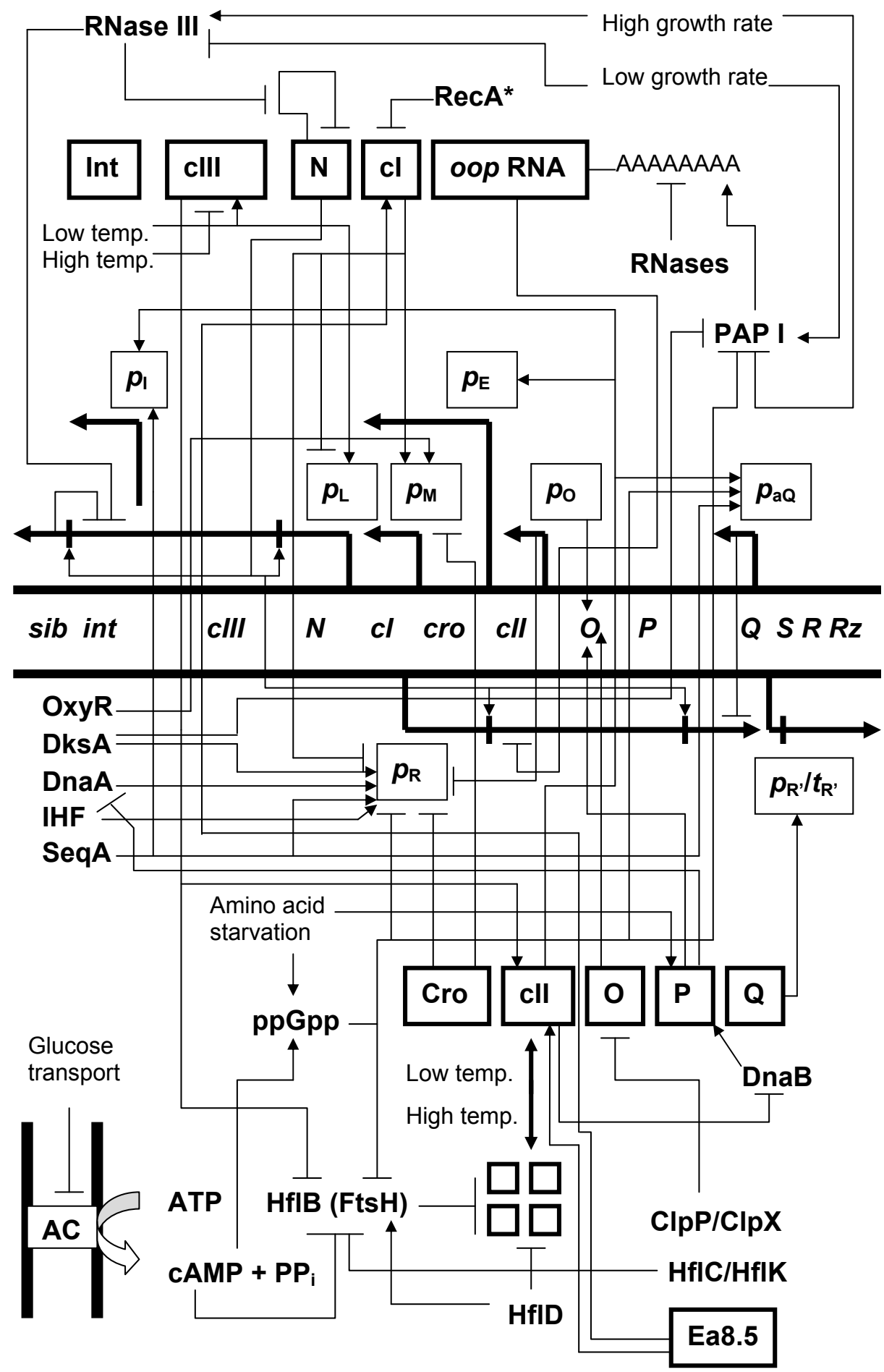

AC - adenylate cyclase; ATP - adenosine triphosphate; cAMP - cyclic AMP; High temp. - high temperature; Low temp. - low temperature; PAP I - poly (A) polymerase I (the pcnB gene product); ppGpp - guanosine tetraphosphate; PPi - pyrophosphate

Figure 1. A regulatory network at the 'lysis-versus-lysogenization' decision of bacteriophage $\lambda$ as an example for the genetic control of a relatively simple biological decision. This decision may represent bacteriophage "personality" (as a metaphor, though with a common biological, but not psychological, sense). The crucial $\lambda$ regulatory genes are presented between two thick horizontal lines that symbolize a fragment of the phage genome. Promoters are marked in thin boxes, and transcripts are shown as thick arrows, with arrowheads indicating directionality of transcription (oop RNA is an exception, see below). Phage $\lambda$ gene products (proteins and one of the non-translatable transcripts, oop RNA) are marked in thick boxes.

The host (Escherichia coli) proteins and specific conditions are presented without boxes. Regulatory processes are indicated as thin arrows and thin blunt-ended lines (positive regulations are represented by arrows and negative regulations are represented by blunt-ended lines).

This figure is an updated version of the scheme published by Węgrzyn, Licznerska, and Węgrzyn (2012), with included data reported by Bloch et al. (2013), Kwan et al. (2013), and Olszewski et al. (2014). 
Because of the complexity of the biological regulation of human personality, interpretation of any experimental results is very complicated. When looking at the bacteriophage $\lambda$ regulatory network, it is worth noting that mutation in any of the genes depicted in Figure 1 results in a significant change in the probability of choosing one of the two alternative options. Moreover, dysfunctions of various genes may give the same final result, i.e. preference of one particular developmental pathway. Therefore, when extrapolating this to the human system, one may conclude that observation of correlations between some genetic variants and certain personality traits does not necessarily tell us about the role of a particular gene in human personality. This is especially pronounced when molecular mechanisms and specific regulatory networks are not recognized yet, as in the case of personality.

\section{FURTHER PERSPECTIVES}

Recently, several articles were published (see below) which tried to summarize our current stage of understanding of the genetic control of personality, and to suggest possibilities for further studies. From the analysis of the literature, it is clear that most information on effects of genes on human personality comes from association studies (Buades-Rotger \& Gallardo-Pujol, 2014, and references therein). Briefly speaking, this kind of investigation is based on advanced statistical analysis of correlations between occurrence of particular alleles of tested genes and selected features of organisms (for example, human personality traits). Such studies may provide many interesting data, especially if they are performed by testing large populations of analyzed organisms. However, when considering molecular mechanisms of investigated processes, association studies can give only preliminary results. Without detailed knowledge about specific effects of a particular allele (genetic polymorphism) on the level and activity of the gene product, any conclusions about involvement of the tested gene in regulation of the studied process can be only speculative. Therefore, in the case of any candidate for a gene of regulatory functions in personality, detailed molecular analysis is necessary to elucidate its exact role, as well as specific effects of particular mutations.

Another methodological problem arising in studies with genetics of human personality is that some mutations associated with particular traits affect efficiency of expression of particular genes rather than the structure and function of gene products. Nucleotide changes in promoter regions, causing modulation in gene transcription efficiency, are classical examples. The problem is that the vast majority of genetic studies are based on testing nucleic acids isolat- ed from blood or other somatic tissues. However, for personality, the processes occurring in neurons are crucial. Therefore, to demonstrate (not only to suggest) functions of certain genes in personality on the basis of associations of mutations in DNA regions responsible for regulation of gene expression, it would be necessary to provide evidence for changes in gene expression in neurons, particularly those included in the brain. Because of the well-known phenomenon of tissue-specific expression of genes, effects in one kind of cell may not necessarily be evident in other kinds of cells. Since studies on expression of genes in the human brain are subject to obvious restrictions, this problem may be very difficult to solve. One possibility is to use animal models, but this kind of experiment also has limitations, especially in studies on personality (discussed above).

The above identified problems are perhaps the basis of a lack of clear evidence for the contribution of specific genetic variants to observed variation in personality traits, indicated by Munafò and Flint (2011). These authors also linked the difficulties in obtaining clear results to the very small effects on personality conferred by individual alleles. This arises from the fact that genetic regulation of personality is likely to consist of the combined effects of thousands of genes, each having only a small effect. Therefore, Munafò and Flint (2011) recognized that statistical stringency, very large sample sizes, and independent replication are crucial points in further studies.

Balestri, Calati, Serretti, and De Ronchi (2014) analyzed published reports describing associations between genes and several personality traits (anxiety, impulsivity, determination-activity, socialization, and spirituality). Importantly, they found no clear consensus on the role of any individual gene variant in personality modulation. These authors suggest (similarly to Munafò \& Flint, 2011) that the combined influence of many genes of small effects on personality may be the reason for the inconsistency found in the literature. They also recommended the use of larger sample sizes and analysis of more narrow and specific phenotypes in further studies.

Contrary to the suggestions summarized in the last two paragraphs, Montag and Reuter (2014) proposed focusing on animal research-based testing of candidate genes, and the use of a priori genotyping (to increase statistical power) in future studies on genetics of personality. They also underlined the importance of integrating cross-cultural data and epigenetic measures in forthcoming personality research. Interestingly, some new tools, useful in such studies, have been proposed, such as the Affective Neuroscience Personality Scales (Montag \& Reuter, 2014).

Finally, Tekkalaki, Tripathi, and Trivedi (2014) proposed to integrate biological and psychological approaches in research devoted to understand mech- 
anisms of personality. They were aware of a growing tendency to neglect psychological aspects in studies on genetic bases of personality, and suggested that biological and psychological studies should be complementary rather than mutually exclusive.

In summary, because of our knowledge on biological mechanisms of development of the central nervous system (for a recent reviews see: Florio \& Huttner, 2014; Yuan \& Hassan, 2014), it appears obvious that our genes must influence our personality, at least at the stage of formation of the network of neurons in the brain which makes predispositions to particular traits; they can be significantly modified then by environmental factors to express actual personality. However, personality is an extremely complex feature, and thousands of genes (each with a relatively small impact) are involved in its formation and regulation. Therefore, elucidation of all the regulatory mechanisms controlled by genes and operating in this complicated system is a real challenge for researchers. It is likely that novel molecular approaches, including rapid sequencing, genomics, transcriptomics, proteomics, and others, will be very helpful in identifying involvement of particular genes in personality traits. Nevertheless, determination of molecular details of specific reactions appears to be necessary to obtain the whole picture, which will perhaps be impossible without simultaneous integration of knowledge from various disciplines, such as genetics and psychology.

\section{REFERENCES}

Amad, A., Ramoz, N., Thomas, P., Jardri, R., \& Gorwood, P. (2014). Genetics of borderline personality disorder: systematic review and proposal of an integrative model. Neuroscience \& Biobehavioral Reviews, 40, 6-19.

Balestri, M., Calati, R., Serretti, A., \& De Ronchi, D. (2014). Genetic modulation of personality traits: a systematic review of the literature. International Clinical Psychopharmacology, 29, 1-15.

Bellettato, C. M., \& Scarpa, M. (2010). Pathophysiology of neuropathic lysosomal storage disorders. Journal of Inherited Metabolic Disease, 33, 347-362.

Bloch, S., Nejman-Faleńczyk, B., Łoś, J. M., Barańska, S., Łepek, K., Felczykowska, A., Łoś, M., Węgrzyn G., $\&$ Węgrzyn, A. (2013). Genes from the exo-xis region of $\lambda$ and Shiga toxin-converting bacteriophages influence lysogenization and prophage induction. Archives of Microbiology, 195, 693-703.

Buades-Rotger, M., \& Gallardo-Pujol, D. (2014). The role of the monoamine oxidase $A$ gene in moderating the response to adversity and associated antisocial behavior: a review. Psychology Research and Behavior Management, 7, 185-200.
Calati, R., Signorelli, M. S., Gressier, F., Bianchini, O., Porcelli, S., Comings, D. E., De Girolamo, G., Aguglia, E., MacMurray, J., \& Serretti, A. (2014). Modulation of a number of genes on personality traits in a sample of healthy subjects. Neuroscience Letters, 566, 320-325.

Caspi, A., McClay, J., Moffitt, T. E., Mill, J., Martin, J., Craig, I. W., Taylor, A., \& Poulton, R. (2002). Role of genotype in the cycle of violence in maltreated children. Science, 297, 851-854.

Caspi, A., Sugden, K., Moffitt,T.E., Taylor, A., Craig, I.W., Harrington, H., McClay, J., Mill, J., Martin, J., Braithwaite, A., \& Poulton, R. (2003). Influence of life stress on depression: moderation by a polymorphism in the 5-HTT gene. Science, 301, 386-389.

Cicchetti, D., Rogosch, F. A., Hecht, K. F., Crick, N. R., \& Hetzel, S. (2014). Moderation of maltreatment effects on childhood borderline personality symptoms by gender and oxytocin receptor and FK506 binding protein 5 genes. Development and Psychopathology, 26, 831-849.

Florio, M., \& Huttner, W. B. (2014). Neural progenitors, neurogenesis and the evolution of the neocortex. Development, 141, 2182-2194.

Glaser, Y. G., Zubieta, J. K., Hsu D. T., Villafuerte, S., Mickey, B. J., Trucco, E. M., Burmeister, M., Zucker, R. A., \& Heitzeg, M. M. (2014). Indirect effect of corticotropin-releasing hormone receptor 1 gene variation on negative emotionality and alcohol use via right ventrolateral prefrontal cortex. The Journal of Neuroscience, 34, 4099-4107.

Hammen, C., Bower, J. E., \& Cole, S. W. (in press). Oxytocin receptor gene variation and differential susceptibility to family environment in predicting youth borderline symptoms. Journal of Personality Disorders. DOI: 10.1521/pedi_2014_28_152

Jakóbkiewicz-Banecka, J., Gabig-Cimińska, M., Banecka-Majkutewicz, Z., Banecki, B., Węgrzyn, A., \& Węgrzyn, G. (2014). Factors and processes modulating phenotypes in neuronopathic lysosomal storage diseases. Metabolic Brain Disease, 29, 1-8.

Kwan, J. J., Smirnova, E., Khazai, S., Evanics, F., Maxwell, K. L., \& Donaldson, L. W. (2013). The solution structures of two prophage homologues of the bacteriophage $\lambda$ Ea8.5 protein reveal a newly discovered hybrid homeodomain/zinc-finger fold. Biochemistry, 52, 3612-3614.

Laplana, M., Royo, J. L., García, L. F., Aluja, A., Gomez-Skarmeta, J. L., \& Fibla, J. (in press). SIRPB1 copy-number polymorphism as candidate quantitative trait locus for impulsive-disinhibited personality. Genes, Brain and Behavior. DOI: 10.1111/ gbb. 12154

Martín-Blanco,A., Ferrer,M.,Soler,J.,Salazar,J.,Vega,D., Andión, O., Sanchez-Mora, C., Arranz, M. J., Ribases, M., Feliu-Soler, A., Pérez, V., \& Pascual, J. C. (2014). Association between methylation of the glucocorticoid receptor gene, childhood maltreat- 
ment, and clinical severity in borderline personality disorder. Journal of Psychiatric Research, 57, 34-40.

Montag, C., \& Reuter, M. (2014). Disentangling the molecular genetic basis of personality: from monoamines to neuropeptides. Neuroscience \& Biobehavioral Reviews, 43, 228-239.

Montag, C., Kunz, L., Axmacher, N., Sariyska, R., Lachmann, B., \& Reuter, M. (2014). Common genetic variation of the APOE gene and personality. BMC Neuroscience, 15, 64.

Munafò, M. R., \& Flint, J. (2011). Dissecting the genetic architecture of human personality. Trends in Cognitive Sciences, 15, 395-400.

Napolioni, V., Murray, D. R., Comings, D. E., Peters, W. R., Gade-Andavolu, R., \& MacMurray, J. (2014). Interaction between infectious diseases and personality traits: $\mathrm{ACP} 1{ }^{*} \mathrm{C}$ as a potential mediator. Infection, Genetics and Evolution, 26, 267-273.

Olszewski, P., Szambowska, A., Barańska, S., Narajczyk, M., Węgrzyn, G., \& Glinkowska, M. (2014). A dual promoter system regulating $\lambda$ DNA replication initiation. Nucleic Acids Research, 42, 44504462.

Schwartz, N., Temkin, P., Jurado, S., Lim, B. K., Heifets, B. D., Polepalli, J. S., \& Malenka, R. C. (2014). Chronic pain. Decreased motivation during chronic pain requires long-term depression in the nucleus accumbens. Science, 345, 535-542.

Tekkalaki, B., Tripathi, A., \& Trivedi, J. K. (2014). Welcome biological breakthroughs, supply psychosocial insights. Mens Sana Monographs, 12, 79-91.

Węgrzyn, G., Jakóbkiewicz-Banecka, J., Narajczyk, M., Wiśniewski, A., Piotrowska, E., Gabig-Cimińska, M., Kloska, A., Słominska-Wojewodzka, M., Korzon-Burakowska, A., \& Węgrzyn, A. (2010). Why are behaviors of children suffering from various neuronopathic types of mucopolysaccharidoses different? Medical Hypotheses, 75, 605-609.

Węgrzyn, G., Licznerska, K., \& Węgrzyn, A. (2012). Phage $\lambda$ - new insights into regulatory circuits. Advances in Virus Research, 82, 155-178.

Wraith, J. E. (2013). Mucopolysaccharidoses and mucolipidoses. Handbook of Clinical Neurology, 113, 1723-1729.

Yuan, L., \& Hassan, B. A. (2014). Neurogenins in brain development and disease: an overview. Archives of Biochemistry and Biophysics, 58, 10-13. 